\title{
THE NONUNIFORM MOTION OF A SUPERSONIC DISLOCATION*
}

\author{
BY \\ CONSTANTINE CALLIAS (Massachusetts Institute of Technology) \\ AND XANTHIPPI MARKENSCOFF (Carnegie-Mellon University)
}

Introduction. The nonuniform motion of a subsonic dislocation has been analyzed previously $[1,2]$. Here we treat the transient motion of a screw supersonic dislocation with particular attention to the wave-front analysis and the detailed treatment of the singularities involved. Although dislocations may not actually move at supersonic speeds, the solution for a dislocation may be used in other problems involving cracks, slip or separation, as in $[3,4$, etc.]. The analysis presented here may also bear on the treatment of problems involving moving singularities in general $[5,6]$.

Consider a screw dislocation parallel to the y-axis with the discontinuity in the displacement component $u_{y}$ across $z=0$ being denoted by $\Delta u$. The dislocation is at rest in an infinite space until time $t=0$ when it begins to move according to $x=l(t)$ (or $t=\eta(x)$, equivalently). It may be easily seen that the problem is equivalent to the one in the halfspace occupying the region $z>0$ (Fig. 1) with the nonzero displacement component satisfying the equation

$$
\frac{\partial^{2} u_{y}}{\partial x^{2}}+\frac{\partial^{2} u_{y}}{\partial z^{2}}=b^{2} \frac{\partial^{2} u_{y}}{\partial t^{2}}
$$

and the boundary conditions at $z=0$

$$
\begin{array}{ll}
u_{y}(x, 0, t)=\frac{1}{2} \Delta u H(x), & t<0, \\
u_{y}(x, 0, t)=\frac{1}{2} \Delta u H(x-l(t)), & t \geq 0,
\end{array}
$$

where $b$ denotes the shear-wave slowness and $H$ the Heaviside step function.

The solution to the above problem is equivalent to the superposition of the following two problems satisfying the equation of motion and the boundary condition:

Problem I: $\quad u_{y}(x, 0)=,\frac{1}{2} \Delta u H(x)$ for all $t ;$

Problem II: $u_{y}(x, 0, t)=\frac{1}{2}[\Delta u H(x-l(t))-\Delta u H(x)]$ for $t \geq 0$,

the solution to the static problem I being known [2]. The subscript $y$ in $u_{y}$ will be deleted subsequently for the sake of simplicity. Also the functions $l(t)$ or $\eta(x)$ are assumed to be monotonically increasing or decreasing, respectively.

The solution to problem II has been obtained in [2] by means of Laplace transforms and inversion by the Cagniard-de Hoop technique. Solving for the strain of interest

* Received November 2, 1979; revised version received December 15, 1979. 
$\partial u / \partial z$, it was derived in [1] (Eq. (32)) that

$$
\begin{aligned}
\frac{\partial u}{\partial z}(x, z, t)= & -\frac{\Delta u}{2 \pi} \int_{0}^{\infty} \frac{(t-\eta(\xi))(x-\xi)^{2} H(t-\eta(\xi)-r b)}{r^{4}\left[(t-\eta(\xi))^{2}-r^{2} b^{2}\right]^{1 / 2}} d \xi \\
& +\frac{\Delta u}{2 \pi} z^{2} \frac{\partial}{\partial t} \int_{0}^{\infty} \frac{(t-\eta(\xi))^{2} H(t-\eta(\xi)-r b)}{r^{4}\left[(t-\eta(\xi))^{2}-r^{2} b^{2}\right]^{1 / 2}} d \xi
\end{aligned}
$$

where $r^{2}=(x-\xi)^{2}+z^{2}$.

Since no assumption about the velocity of the dislocation was made in the derivation of the above equation, this expression holds both for subsonic and supersonic motion. However, the nature of the solution is very different in the two cases because of the different behavior of the argument $f(\xi)=t-\eta(\xi)-r b$ of the step function which defines the limits of integration and the different limiting behavior of the integrals. This behavior is analyzed in what follows, after the treatment of the constant-velocity case; this is presented first in order to illustrate the basic differences between the subsonic and supersonic motion.

1. Dislocation starting from rest and moving with constant velocity. By direct formulation of the problem as a constant-velocity one, the solution may be obtained by a procedure similar to that in [2], with the delta function due to the fact that in the supersonic case a pole is included in the inversion contour:

$$
\begin{aligned}
& \frac{\partial u}{\partial z}(x, z, t) \\
& =\frac{\Delta u}{2 \pi} \begin{cases}\frac{-x}{x^{2}+z^{2}} & \text { for } t \leq r b \\
\frac{-x}{x^{2}+z^{2}}+\frac{\left[b^{2}-\frac{t^{2} x^{2}}{r_{0}^{4}}+\frac{z^{2}}{r_{0}^{4}}\left(t^{2}-r_{0}^{2} b^{2}\right)\right]\left(\alpha-\frac{t x}{r_{0}^{2}}\right)+\frac{2 t x}{r_{0}^{6}} z^{2}\left(t^{2}-r_{0}^{2} b^{2}\right)}{\sqrt{t^{2}-r_{0}^{2} b^{2}}\left[\left(\alpha-\frac{t x}{r_{0}^{2}}\right)^{2}+\frac{z^{2}\left(t^{2}-r_{0}^{2} b^{2}\right.}{r_{0}^{4}}\right]} & \text { for } t \geq r b\end{cases}
\end{aligned}
$$

$$
+\frac{\Delta u}{2} \sqrt{b^{2}-\alpha^{2}} \delta\left(t-\alpha x \mp \sqrt{b^{2}-\alpha^{2}} z\right)(- \text { for } z>0 ;+ \text { for } z<0)
$$

where $r^{2}=x^{2}+z^{2}$. The argument of the delta function corresponds to the shock-wave front which consists of the envelope of the wavelets that were excited by the dislocation on its way (Fig. 1). It is worth noting that behind the wave-front the stress field at a point receives contributions from two source points and that an interference mechanism results in the vanishing of the disturbances for the case of the constant-velocity motion. This does not occur, however, if the dislocation is moving with variable velocity, as in the case discussed next.

2. Wave-front analysis for a nonuniformly moving dislocation. In order to study the wave-front behavior of the solution (1), we have to look closer at the behavior of the integral, and first at the form of the function $f(\xi)=t-\eta(\xi)-r b$ which defines the intervals of the integration. For the constant-velocity case, $f(\xi)$ is plotted in Fig. 2(a) 


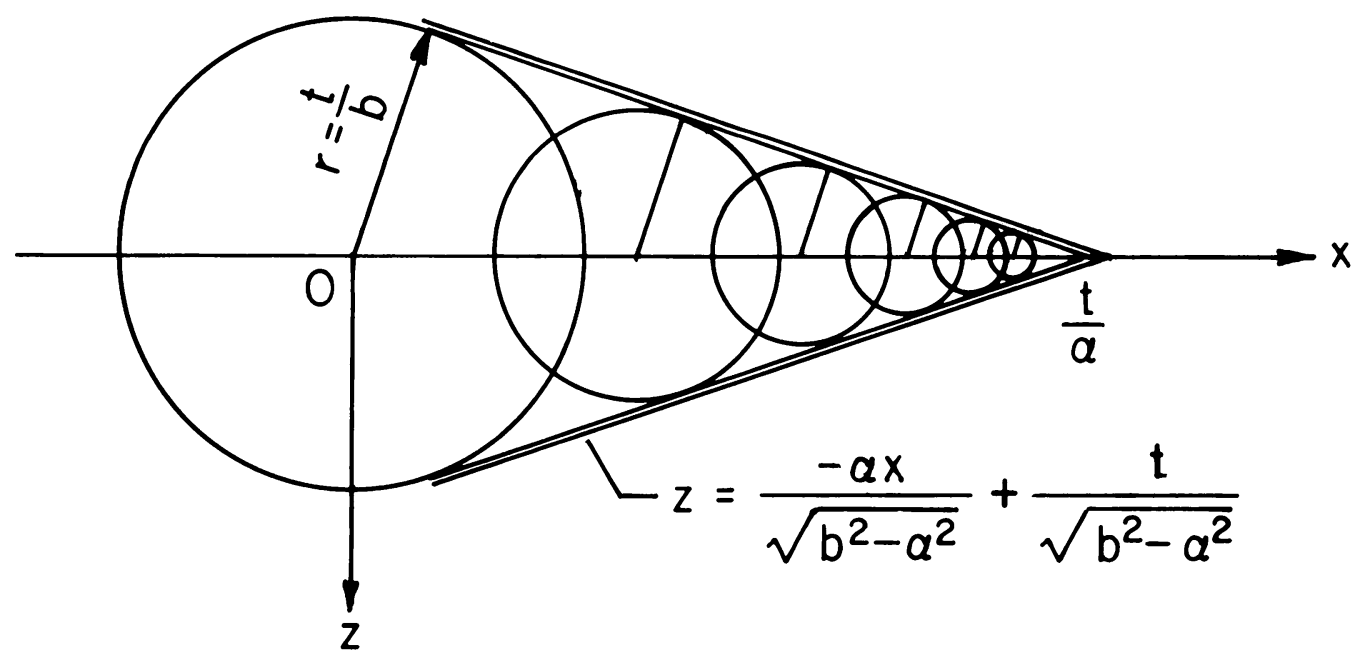

FIG. 1.

and 2(b) for supersonic and subsonic motion, respectively. For subsonic motion the lower limit of integration is always zero, while for supersonic motion the interval of the integration may not contain the origin and $f(\xi)$ exhibits a maximum in the range of integration. For a particular combination of $t, x, z$, the range of integration vanishes and it may be easily seen that this particular combination corresponds to the wave-front $t=\alpha x+\left(b^{2}-\alpha^{2}\right)^{1 / 2} z$.

While for a constant-velocity motion the curve $f(\xi)=t-\alpha \xi-r b$ has one maximum, for a general motion $\eta(\xi)$ the function $f(\xi)$ may exhibit more than one maximum. If $\eta^{\prime}(\xi)$ increases monotonically then $f(\xi)$ will have only one maximum and the behavior of $f(\xi)$ is similar to the constant-velocity motion, although the wave-fronts are no longer straight lines (Fig. 3a). If $\eta^{*}(\xi)$ is a decreasing function in some intervals, then $f(\xi)$ will also have a minimum for some $t, x, z$ (Fig. $3 \mathrm{~b}$ ) while for suitable $(t, x, z), f(\xi)$ can also have more than one maximum (Fig. 3c). The appearance of cusps in the wave-fronts has been discussed by Freund [5] in the analysis of wave-front behavior by a different approach.

Let us consider the instance in which the range of integration tends to zero, i.e., $\xi_{0}$, $\xi_{1} \rightarrow \xi_{*}$ (Fig. 4). If $f(\xi)$ has a maximum in $\left[\xi_{0}, \xi_{1}\right]$, this will always happen for $t_{*}$ and $\xi_{*}$ that satisfy:

$$
\begin{array}{r}
f\left(\xi_{*}\right)=t_{*}-\eta\left(\xi_{*}\right)-\sqrt{\left(x-\xi_{*}\right)^{2}}+z^{2} b=0, \\
\frac{d}{d \xi}\left(t_{*}-\eta\left(\xi_{x}\right)-\sqrt{\left(x-\xi_{*}\right)^{2}+z^{2}} b\right)=0 .
\end{array}
$$

The wave-front equation can be derived by elimination of $\xi_{*}$ between (3) and (4). (The curve $f\left(\xi_{*}\right)$ obviously should correspond to the wave-front since it separates regions of zero and nonzero range of integration.) Eq. (3) and (4) can also be obtained if the wave-front is seen as the envelope of the wavelets with equation

$$
(x-l(t))^{2}+z^{2}=\frac{1}{b^{2}}(t-\tau)^{2} .
$$




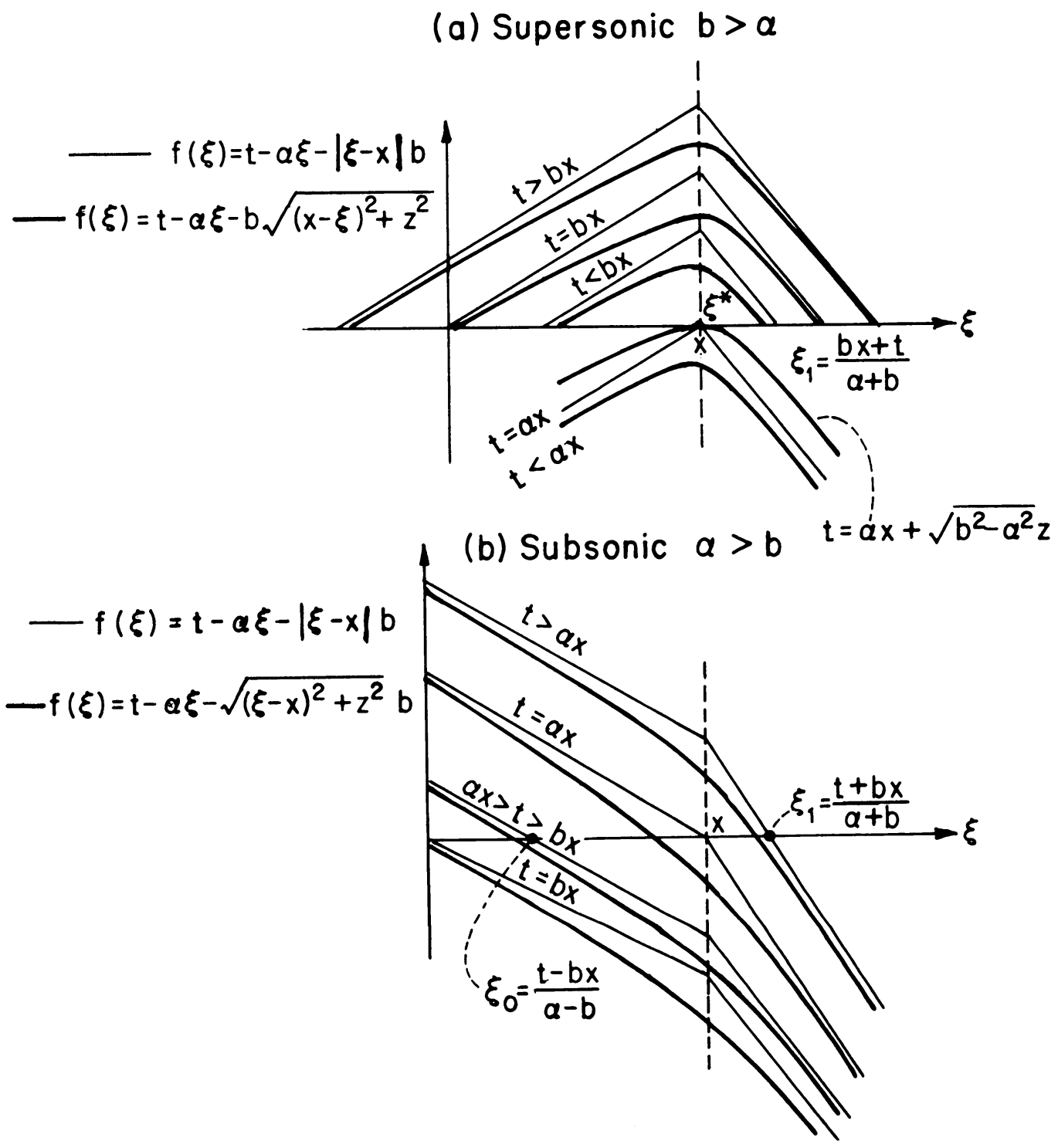

FIG. 2.

Each of the two integrals appearing in the general equation for the stress can be stedied asymptotically as we approach the wave-front in a systematic way. Since we are near the wave-front we can expand in $t-t_{*}$. On the other hand, since in the limit the range of the integral becomes small, we can expand the intergrand in $\xi-\xi_{*}$. The result is

$$
\begin{aligned}
(t-\eta(\xi))^{2}-r b^{2}= & \left(\eta^{\prime}\left(\xi_{*}\right)^{2}-b^{2}\right)\left(\xi-\xi_{*}\right)^{2} \\
& +2\left(t_{*}-\eta\left(\xi_{*}\right)\right)^{2}\left(t-t_{*}\right)+\left(t-t_{*}\right)^{2}-2\left(t-t_{*}\right) \eta^{\prime}\left(\xi_{*}\right)\left(\xi-\xi_{*}\right) \\
& + \text { higher-order terms in }\left(\xi-\xi_{*}, t-t_{*}\right) .
\end{aligned}
$$




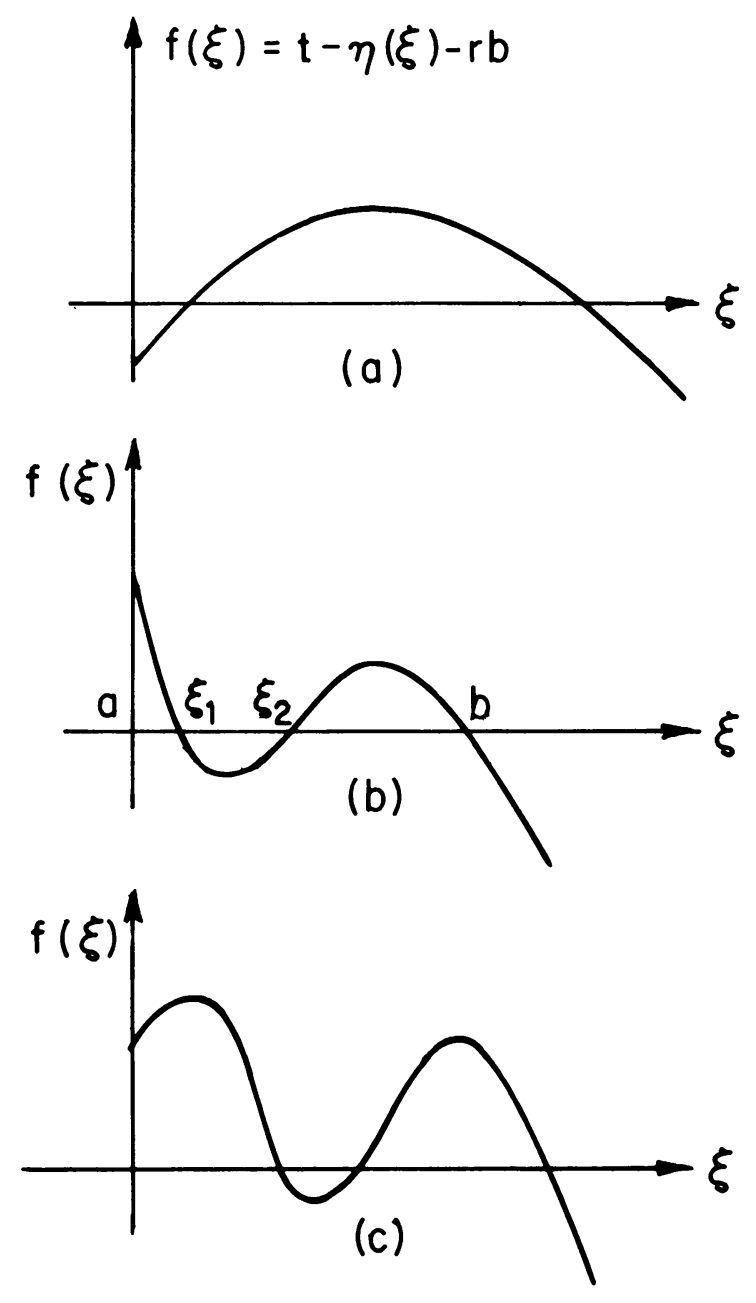

FIG. 3.

The roots of this expression give $\xi_{0}, \xi_{1}$ in the asymptotic limit, so we write it as

$$
-\left(\eta^{\prime}\left(\xi_{*}\right)^{2}-b^{2}\right)\left(\xi-\xi_{0}\right)\left(\xi_{1}-\xi\right) .
$$

Thus to leading order the term in (1) that gives rise to the $\delta$-function, i.e. the second term, can be written

$$
\frac{\Delta u}{2 \pi} z^{2} \frac{\partial}{\partial t} \int_{\xi_{0}}^{\xi_{1}} \frac{\left(t_{*}-\eta\left(\xi_{*}\right)\right)^{2} H\left(t-t_{*}\right) d \xi}{\left[\left(\xi_{*}-x\right)^{2}+z^{2}\right]^{2}\left[b^{2}-\eta^{\prime}\left(\xi_{*}\right)^{2}\right]^{1 / 2} \sqrt{\left(\xi-\xi_{0}\right)\left(\xi_{1}-\xi\right)}},
$$

where the step function $H\left(t-t_{*}\right)$ indicates that the integral vanishes for $t<t_{*}$ (outside the wave-front). To leading order in $\left(t-t_{*}\right),(5)$ obviously gives

where

$$
A \delta\left(t-t_{*}\right)
$$

$$
A=\lim _{\xi_{0}, \xi_{1} \rightarrow \xi_{*}} \frac{\Delta u}{2 \pi} z^{2} \frac{\left(t_{*}-\eta\left(\xi_{*}\right)\right)^{2}}{\left[\left(\xi_{*}-x\right)^{2}+z^{2}\right]^{2}\left[b^{2}-\eta^{\prime}\left(\xi_{*}\right)^{2}\right]^{1 / 2}} \int_{\xi_{0}}^{\xi_{1}} \frac{d \xi}{\sqrt{\left(\xi-\xi_{0}\right)\left(\xi_{1}-\xi\right)}} .
$$




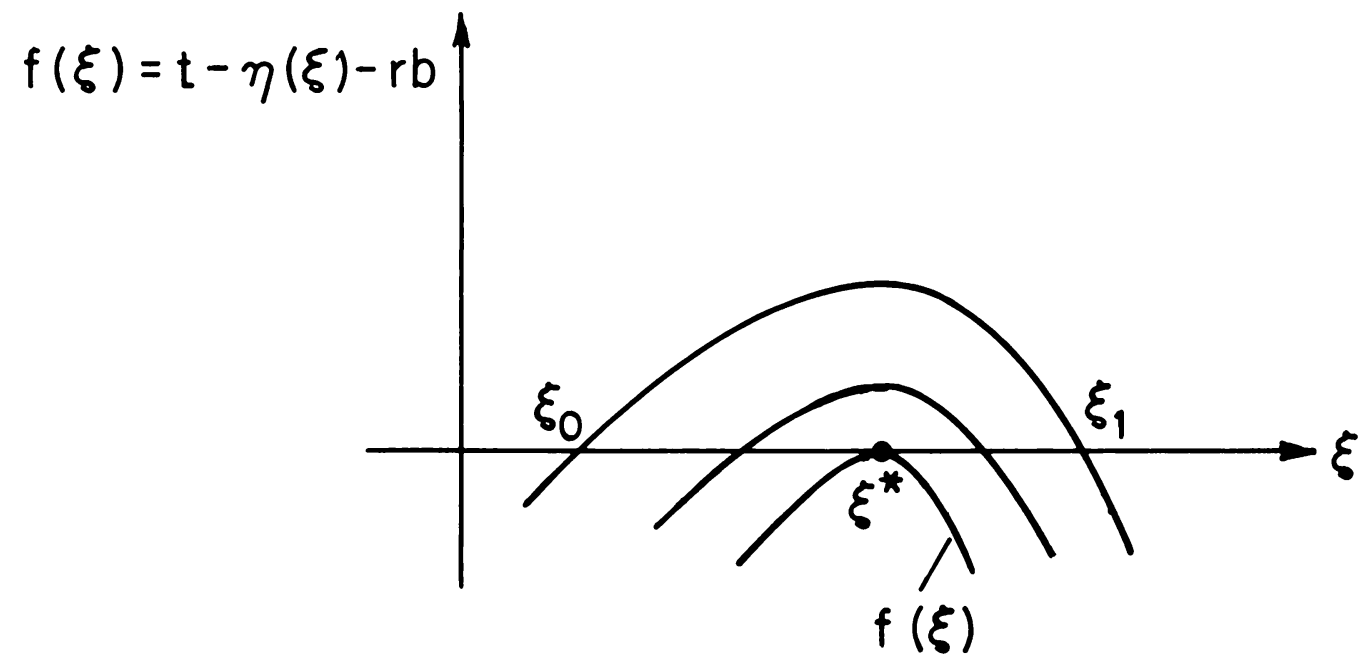

FIG. 4.

The integral has the value $\pi$ and we find that

$$
A=\frac{\Delta u}{2} \cdot z^{2} \frac{\left(t_{*}-\eta\left(\xi_{*}\right)\right)^{2}}{\left[\left(\xi_{*}-x\right)^{2}+z^{2}\right]^{2} \cdot\left[b^{2}-\eta^{\prime}\left(\xi_{*}\right)^{2}\right]^{1 / 2}} .
$$

We study now the behavior of the solution near a minimum of $f(\xi)$ tangent to the $\xi$-axis, i.e. as $\xi_{1}, \xi_{2} \rightarrow \xi^{*}$ in Fig. 3(b). To study qualitatively the behavior of the solution near such a minimum we look at the behavior of the integral

$$
\int_{a}^{b} \frac{H\left(\left(\xi-\xi^{*}\right)+\varepsilon(t)\right)}{\sqrt{\left(\xi-\xi^{*}\right)^{2}}+\varepsilon} d \xi
$$

This is not an approximation to our integrands but it simulates the behavior of the integrands near the roots of the argument of the step function. For $\varepsilon<0$, (6) gives:

$$
\begin{aligned}
& \int_{a}^{\xi *-\sqrt{ }-\varepsilon} \frac{d \xi}{\left[\left(\xi-\xi^{*}\right)^{2}+\varepsilon\right]^{1 / 2}}+\int_{\xi^{*+}-\varepsilon}^{b} \frac{d \xi}{\left[\left(\xi-\xi^{*}\right)^{2}+\varepsilon\right]^{1 / 2}} \\
&=\cosh ^{-1} \frac{\xi^{*}-a}{\sqrt{-\varepsilon}}+\cosh ^{-1} \frac{b-\xi^{*}}{\sqrt{-\varepsilon}}=\ln \left(\frac{\xi^{*}-a}{\sqrt{-\varepsilon}}+\sqrt{\frac{\left(\xi^{*}-a\right)^{2}}{-\varepsilon}-1}\right. \\
&+\ln \left(\frac{b-\xi^{*}}{\sqrt{-\varepsilon}}+\sqrt{\frac{\left(b-\xi^{*}\right)^{2}}{-\varepsilon}}-1\right),
\end{aligned}
$$

since the interval $\left(\xi^{*}-\sqrt{ }-\varepsilon, \xi^{*}+\sqrt{ }-\varepsilon\right)$ obviously does not belong to the range of integration in this case. On the other hand, for $\varepsilon>0$ we have

$$
\begin{aligned}
\int_{a}^{b} \frac{d \xi}{\left[\left(\xi-\xi^{*}\right)^{2}+\varepsilon\right]^{1 / 2}} & =\left.\sinh ^{-1} \frac{\xi-\xi^{*}}{\sqrt{\varepsilon}}\right|_{a} ^{b} \\
& =\ln \left(\frac{b-\xi^{*}}{\sqrt{\varepsilon}}+\sqrt{\frac{\left(b-\xi^{*}\right)^{2}}{\varepsilon}+1}\right)-\ln \left(\frac{a-\xi^{*}}{\sqrt{\varepsilon}}+\sqrt{\frac{\left(a-\xi^{*}\right)^{2}}{\varepsilon}+1}\right)
\end{aligned}
$$


and it may be noticed that both (7) and (8) are equal to

$$
\ln \frac{\left(b-\xi^{*}\right)+\sqrt{\left(b-\xi^{*}\right)^{2}+\varepsilon}}{\xi^{*}-a+\sqrt{\left(\xi^{*}-a\right)^{2}+\varepsilon}}
$$

for $\varepsilon<0$ and $>0$ respectively, so that the second integral in (1) is a continuous function as we approach the wave-front and therefore the time derivative cannot contain singularities of the delta-function type.

3. Solution away from the wave-front. The solution to the general motion of a supersonic dislocation is given by Eq. (1). However, in the second integral differentiation with respect to $t$ inside the integral is not permissible, since then the integral will obviously diverge. With proper manipulations and integration by parts-which we show below-it can be brought to a suitable form for differentiating inside.

Consider the range of integration $\left[\xi_{1}, \xi_{2}\right]$ between two consecutive real roots $\xi_{1}$ and $\xi_{2}$ of $t-\eta(\xi)-\left((x-\xi)^{2}+z^{2}\right)^{1 / 2} b=0$. Then we may write:

$$
\begin{aligned}
& \frac{\partial}{\partial t} \int_{\xi_{1}}^{\xi_{2}} \frac{(t-\eta(\xi))^{2} d \xi}{r^{4}\left[(t-\eta(\xi))^{2}-r^{2} b^{2}\right]^{1 / 2}}=\frac{\partial}{\partial t} \int_{\xi_{1}}^{\xi_{2}} \frac{(t-\eta(\xi))^{2}}{r^{4}} \frac{\sqrt{\left(\xi-\xi_{1}\right)\left(\xi_{2}-\xi\right)}}{\sqrt{(t-\eta(\xi))^{2}-r^{2} b^{2}}} \frac{d \xi}{\sqrt{\left(\xi-\xi_{1}\right)\left(\xi_{2}-\xi\right)}} \\
&= \frac{\partial}{\partial t} \int_{\xi_{1}}^{\xi_{2}} \frac{(t-\eta(\xi))^{2}}{r^{4}} \frac{\sqrt{\left(\xi-\xi_{1}\right)\left(\xi_{2}-\xi\right)}}{\sqrt{(t-\eta(\xi))^{2}-r^{2} b^{2}}}\left(-d \cos ^{-1} \frac{\xi-\alpha}{\beta}\right) \\
&=\left.\frac{\partial}{\partial t}\left[-\frac{b^{2}}{r^{2}} \sqrt{\frac{\left(\xi-\xi_{1}\right)\left(\xi_{2}-\xi\right)}{(t-\eta(\xi))^{2}-r^{2} b^{2}}}\right]\right|_{\xi=\xi_{1}} ^{\xi=\xi_{2}} \\
& \quad+\frac{\partial}{\partial t} \int_{\xi_{1}}^{\xi_{2}} \cos ^{-1} \frac{\xi-\alpha}{\beta} \frac{\partial}{\partial \xi} \frac{(t-\eta(\xi))^{2}}{r^{4}} \sqrt{\frac{\left(\xi-\xi_{1}\right)\left(\xi_{2}-\xi\right)}{\left.(t-\eta(\xi))^{2}-r^{2} b^{2}\right)}} d \xi
\end{aligned}
$$

where $\left(\xi-\xi_{1}\right)\left(\xi_{2}-\xi\right) \equiv \beta^{2}-(\xi-\alpha)^{2}$. We may remark, in the last integral of (9), that

$$
\sqrt{\frac{\left(\xi-\xi_{1}\right)\left(\xi_{2}-\xi\right)}{(t-\eta(\xi))^{2}-r^{2} b^{2}}}
$$

is regular and

$$
\frac{\partial}{\partial t} \cos ^{-1} \frac{\xi-\alpha}{\beta}=\frac{-1}{\sqrt{\left(\xi-\xi_{1}\right)\left(\xi_{2}-\xi\right)}} \frac{\xi_{2}-\xi_{1}}{2} \frac{\partial}{\partial t} \frac{\xi-\alpha}{\beta},
$$

so that the singularity is now integrable and differention with respect to $t$ may be performed inside the integral. It is also useful to observe that

$$
\frac{\partial \xi_{i}}{\partial t}=1 /\left\{\eta^{\prime}\left(\xi_{i}\right)+\frac{\xi_{i}-x}{r_{i}} b\right\}, \quad i=1,2 .
$$

The stress on the slip plane $z=0$ is frequently of interest. An alternate way of taking the limit as $z \rightarrow 0$ in (1) would be to use the solution for $z=0$ obtained in [2] (Eq. (25)) 
where the constant-velocity solution added and subtracted is now for supersonic motion as in (2). We have for the strain of interest

$$
\begin{aligned}
& \frac{\partial u}{\partial z}(x, 0, t)=\frac{-\Delta u}{2 \pi} \int_{0}^{\infty} \frac{(t-\eta(\xi)) H(t-\eta(\xi)-|x-\xi| b) d \xi}{\sqrt{(t-\eta(\xi))^{2}-(x-\xi)^{2} b^{2}}(x-\xi)^{2}} \\
& -\frac{\Delta u}{2 \pi} \int_{0}^{\infty} \frac{\left((t-\eta(x))-\eta^{\prime}(x)(\xi-x)\right) H\left(t-\eta(x)-\eta^{\prime}(x)(\xi-x)-|\xi-x| b\right)}{\sqrt{\left(t-\eta(x)-\eta^{\prime}(x)(\xi-x)\right)^{2}-(x-\xi)^{2} b^{2}}(x-\xi)^{2}} \\
& +\frac{\Delta u}{2 \pi} \frac{1}{t-\eta(x)} \sqrt{\left(\frac{t+\eta^{\prime}(x) x-\eta(x)}{x}\right)^{2}-b^{2}} H\left(t-|x| b-\eta(x)+x \eta^{\prime}(x)\right) \\
& +\frac{\Delta u}{2} \sqrt{b^{2}-\eta^{\prime}(x)^{2}} \delta(t-\eta(x))
\end{aligned}
$$

This concludes our analysis of the main features of the general supersonic motion of a screw dislocation. The methodology used here may prove useful for the treatment of the edge dislocation and other moving singularities.

\section{REFERENCES}

[1] J. D. Eshelby, Phys. Rev. 90, 248 (1953)

[2] X. Markenscoff, J. Elast. 10, 193 (1980)

[3] P. Burgers and L. B. Freund, Int. J. Sol. Str. 16, 265 (1980)

[4] S. Winkler, D. R. Curran, and D. A. Shockey, Int. J. Fr. Mech. 6, 151, 271 (1970)

[5] L. B. Freund, Quart. Appl. Math. 30, 271 (1972)

[6] L. M. Brock, Quart. Appl. Math. 36, 269 (1978) 